Original Research Paper

\title{
Deficient Reasoning for Dark Matter in Galaxies
}

\author{
James Q. Feng and C.F. Gallo \\ Superconix, Inc., 2440 Lisbon Avenue, Lake Elmo, MN 55042, USA
}

Article history

Received: 06-10-2014

Revised: 08-01-2015

Accepted: 14-01-2015

Corresponding Author:

James Q. Feng

Superconix, Inc., 2440 Lisbon

Avenue, Lake Elmo,

MN 55042, USA

Email: james.q.feng@gmail.com

\begin{abstract}
In this universe, not all of the matter around us can be readily seen. The further an object is away from us and the less luminous it is, the less visible it becomes. Just by looking at an object is usually difficult, if not impossible, to tell the amount of mass it contains. But astronomers have been using the measured luminosity to estimate the luminous mass of stars, based on empirically established mass-to-light ratio which seems to be only applicable to a special class of stars---the main-sequence stars---with still considerable uncertainties. Another basic tool for astronomers to determine the mass of a system of stars or galaxies comes from the study of their motion, as Newton demonstrated with his law of gravitation, which yields the gravitational mass. Because the luminous mass can at best only represent a portion of the gravitational mass, finding the luminous mass to be different or less than the gravitational mass should not be surprising. Using such an apparent discrepancy as compelling evidence for the socalled dark matter, which has been believed to possess mysterious nonbaryonic properties having a dominant amount in galaxies and the universe, seems to be too far a stretch when seriously examining the facts and uncertainties in the measurement techniques. In our opinion, a galaxy with star type distribution varying from its center to edge may have a massto-light ratio varying accordingly. With the thin-disk model computations based on measured rotation curves, we found that most galaxies have a typical mass density profile that peaks at the galactic center and decreases rapidly within $\sim 5 \%$ of the cut-off radius and then declines nearly exponentially toward the edge. The predicted mass density in the Galactic disk is reasonably within the reported range of that observed in interstellar medium. This leads us to believe that ordinary baryonic matter can be sufficient for supporting the observed galactic rotation curves; speculation of large amount of non-baryonic matter may be based on an ill-conceived discrepancy between gravitational mass and luminous mass which appears to be unjustified.
\end{abstract}

Keywords: Galaxy, Dark Matter, Luminous Mass, Rotation Curve

\section{The Popular Belief}

In present days, a large number of people would believe, as they have been told, that "dark matter" makes up about $83 \%$ of the universe by mass and is needed to hold the galaxies together. Probably few of them actually made sufficient efforts in researching the validity of the reasons. In fact, serious in-depth discussion of such reasons and validity of which may not even exist in the "reputable" scientific literature, as we have not been able to satisfactorily find so far. To this situation, one might better be reminded that "Smart is when you believe only half of what you hear. Brilliant is when you know which half to believe."
When discussing the dark matter, we should start with its definition. A common description of dark matter suggests that it is a type of matter hypothesized to account for effects that appear as a result of mass where no such mass can be seen. It neither emits nor absorbs electromagnetic radiation (which includes light) at any significant level; it is matter not reactant to light, but its existence and properties are inferred from its gravitational effects on visible matter, radiation and large-scale structure of the universe (Trimble, 1987).

The reason for astrophysicists to hypothesize dark matter seems to be the discrepancies, as they believe according to their findings, between the mass of large astronomical objects determined from their gravitational 
effects and the mass derived from the luminous matter those objects contain (Freeman and McNamara, 2006). From how mass is defined in classical physics, the method for determining mass from its gravitational effect is straightforward to comprehend. Yet, ways of deriving the "visible" or "luminous" mass (from observed stars, gas and dust) as usually quoted in literature do not appear to have been convincingly explained with scientific rigor, though sometimes can be quite convoluted and difficult to follow. Apparently people in astrophysics tend to rush in with results or "evidence" that cannot always stand up to serious scientific scrutiny. Nonetheless, tremendous efforts and resources have been committed to prematurely declared phenomena that attracted a good deal of press attention.

Here we attempt to examine the available evidence for such conceived dark matter, starting with a brief review of common methods for measuring mass. Then, we discuss the results from evaluating the "gravitational mass" and "luminous mass" in galaxies, the findings of discrepancy between the two and the reasoning for consideration of (mysterious) dark matter as well as the deficiencies therewith. We finally reach our conclusions that ordinary baryonic matter, some of which may be dark or difficult to see, could be sufficient for explaining the observed galactic rotation, the discrepancy between gravitational mass and luminous mass, among other phenomena.

\section{Methods for Determining Mass}

In classical physics, mass is defined as a property of an object which determines its resistance to being accelerated by a force and the strength of its mutual gravitational attraction with other objects. As suggested by its definition, the (inertial) mass of an object can be determined from the measured force acting on it and its responsive acceleration, such as from the ratio of force and acceleration according to Newton's second law of motion. This is similar to the measurements of other material properties such as elasticity where the ratio of measured force (or "stress") and responsive deformation (or "strain") are used in the calculation.

Weighing an object to determine its mass is a common technique called gravimetric method, one typical form of which is to use a spring to counteract the force of gravity pulling on the object. In the earth-bound environment, the gravimetric method is probably the most precise and reliable method for measuring mass. Sophisticated highprecision gravimeters have been used to measure density variations in the rocks making up the Earth, to monitor gravity changes due to mass displacements inside the Earth and to define gravity anomalies.

When the force is of gravitational nature, the gravitational field of an object (which is proportional to its mass) can be determined by measuring the free-fall acceleration of a small 'test object' and from its gravitational field, the object's (active gravitational) mass can be determined. For example, a textbook-method for determining the Sun's mass (Bennett et al., 2007) is to apply the formula of Newton's version of Kepler's third law which leads to (by ignoring the Earth's mass comparing to that of the Sun)

$$
M_{\text {Sun }} \approx \frac{4 \pi^{2} a^{3}}{G p^{2}} \approx 2.0 \times 10^{30}(\mathrm{~kg})
$$

where, $a$ is the measured average distance between the Earth and the Sun $\left(\approx 1.5 \times 10^{11} \mathrm{~m}\right), G$ the gravitational constant $\left(=6.67 \times 10^{11} \mathrm{~m}^{3} \mathrm{~kg}^{-1} \mathrm{~s}^{-2}\right)$ (as can be determined by measuring the attraction of two massive objects in a sensitive torsion balance) and $p$ the Earth's orbital period $\left(\approx 3.15 \times 10^{7}\right.$ s, i.e., 1 year $)$. Actually, (1) can be rearranged as

$$
\frac{G M_{\text {Sun }}}{a^{2}} \approx\left(\frac{2 \pi a}{p}\right)^{2} \frac{1}{a}=\frac{V^{2}}{a}
$$

where, the left side is the gravitational field of the Sun (at the Earth's orbit) and right side the centripetal acceleration of the Earth (with $V$ denoting the magnitude of Earth's orbital velocity).

One of the key variables for determining the mass of a celestial object is its distance to a reference position, like $a$ in (1). For some close objects such as the moon, the planets, the stars in the local solar neighborhood, their distances can be measured by stellar parallax either from the Earth or by using the Earth's orbit. Without any assumption about the nature of stars, parallax is the only technique that can determine the distances of stars. But it is only reliable for accurate measurement of stars within a few hundred light years in the local solar neighborhood (Bennett et al., 2007) (in Astronomical Units (AU), the distance of a star in parsec ( $\mathrm{pc}$ ) equals the reciprocal of the parallax angle in arcseconds, i.e., $1 \mathrm{pc}=3.086 \times 10^{16}$ $\mathrm{m} \approx 3.26$ light-years).

Stellar parallax has enabled measurements of distances of more than 300 stars within about 10 (pc) (or $\sim 33$ light-years) of the Sun, among which about half are binary star systems consisting of two orbiting stars or multiple star systems of three or more stars. The binary star systems are very important in astrophysics, because the information of their orbital motion provides opportunity to directly determine masses of their component stars. For example, the sum of the two star's masses can be calculated from Newton's version of Kepler's third law similar to (1), if both their orbital period (which is relatively easier to measure) and the distance between them are known. The individual masses of the two stars can then be determined from their relative velocities around their common center of mass. 
Once a star's distance is measured from parallax, its luminosity can be determined with the inverse square law for light intensity. With the known luminosity and mass of each individual star for an observed binary star system, an empirical mass-tolight relationship can be determined, from which the masses of single stars may be estimated indirectly based on their measured luminosity.

But in reality, it is often rather difficult to determine the average separation between the two stars in a binary system, because $a$ is needed in using a formula like (1). Among all types of binary star systems, only eclipsing binaries of a pair of stars orbiting in the plane of our line of sight allow detailed study of stellar masses.

For stars that do not belong to any of the binary star systems or outside the local solar neighborhood when stellar parallax becomes inapplicable, their masses may only be estimated indirectly from the established value of mass-to-light ratio, which by itself may consist of considerable uncertainties.

Stars can have wide ranges of luminosity, surface temperature and mass. When measured luminosity and surface temperature of stars are plotted in a scatter graph called the Hertzsprung-Russell (H-R) diagram, some correlative patterns seem to exist between the luminosity and surface temperature (or stellar color), especially for the so-called "main-sequence" stars that fit in a continuous distinctive band. All main-sequence stars are fusing hydrogen into helium in their core, just like the Sun. Their differences in luminosity and surface temperature can be a result of their mass-dependent rate of hydrogen fusion, because more mass is required to maintain gravitational equilibrium with the higher rate of nuclear fusion. Therefore, the mass of a main-sequence star may be expected to fall within the same range of other stars of the same spectral type in the H-R diagram. The surface temperature of a star is easier to measure than luminosity, because it is not expected to change with distance. If a star is determined to belong to the main sequence, its luminosity may be inferred from the $\mathrm{H}-\mathrm{R}$ diagram and used as a standard candle, which then enables the calculation of its distance from its apparent brightness based on the inverse square law---a technique known as the main-sequence fitting. (Uncertainty always exists in main-sequence fitting, because no astronomical object is a perfect standard candle; the challenge of finding the objects that can serve as the best standard candles, therefore, directly relates to the challenge of measuring astronomical distances). Since its presentation in the first decade of the twentieth century, the H-R diagram has become one of the most important tools in astronomical research, remaining central to the analysis of luminous stars (Bennett et al., 2007).
However, for objects other than the main-sequence stars, the reported values of their masses have been derived with much less logical clarity and scientific reliability. At the present stage of our knowledge, "because the methods used for studying the amount of matter at different scales are so diverse, there is always the possibility that one or all of the estimates could be wrong" (Freeman and McNamara, 2006).

\section{Mass in a Galaxy}

As a stellar system consisting of a large number $\left(10^{5}---10^{12}\right)$ of gravitationally bound stars, an interstellar medium of gas and cosmic dust, among others, a galaxy has its mass distributed in an extensive space. Observations have shown that many (late-type, mature) spiral galaxies share a common structure with the visible matter distributed in a flat thin disk, rotating about their center of mass in nearly circular orbits (Fig. 1). Many astrophysical systems, such as spiral galaxies, planetary systems, planetary rings, accretion disks, etc., appear flat for a basic reason: the state of lowest energy is a flat disk perpendicular to an axis along which a distribution of angular momentum is given for a system of constant mass (Binney and Tremaine, 2008).

Thus, it may not be unreasonable to consider a galaxy as an axisymmetric thin disk consisting of distributed self-gravitating mass in balance with a distributed centrifugal force due to rotation in circular orbit. In fact, many observations and measurements of galaxies, such as rotation curves, surface brightness, among others, are presented in terms of variables as functions of the galactocentric radius of an axisymmetric circular thin disk. If we approximate a galaxy as a self-gravitating continuum of axisymmetrically distributed mass in a thin disk with an edge at finite radius $R_{g}$, beyond which the mass density is expected to diminish to the inter-galactic level, the gravitational field at a galactocentric radius $r$ can be calculated as

$\frac{G M_{g}}{R_{g}{ }^{2}} \int_{0}^{1}\left[\int_{0}^{2 \pi} \frac{(r-\hat{r} \cos \varphi) d \varphi}{\left(\hat{r}^{2}+r^{2}-2 \hat{r} r \cos \varphi\right)^{3 / 2}}\right] \rho(\hat{r}) h \hat{r} d \hat{r}$

where, all the variables are made dimensionless by measuring length (e.g., $r, \hat{r}, h$ ) in units of the galactic radius $R_{g}$ and mass density $\rho$ in units of $M_{g} / R_{g}{ }^{3}$ with $M_{g}$ denoting the total mass of the galaxy. Here, the disk thickness $h$ is assumed to be constant.

The centripetal acceleration of an object at $r$ rotating with a velocity $V(r)$ can be written as

$\frac{V_{0}^{2}}{R_{g}} \frac{V(r)^{2}}{r}$ 

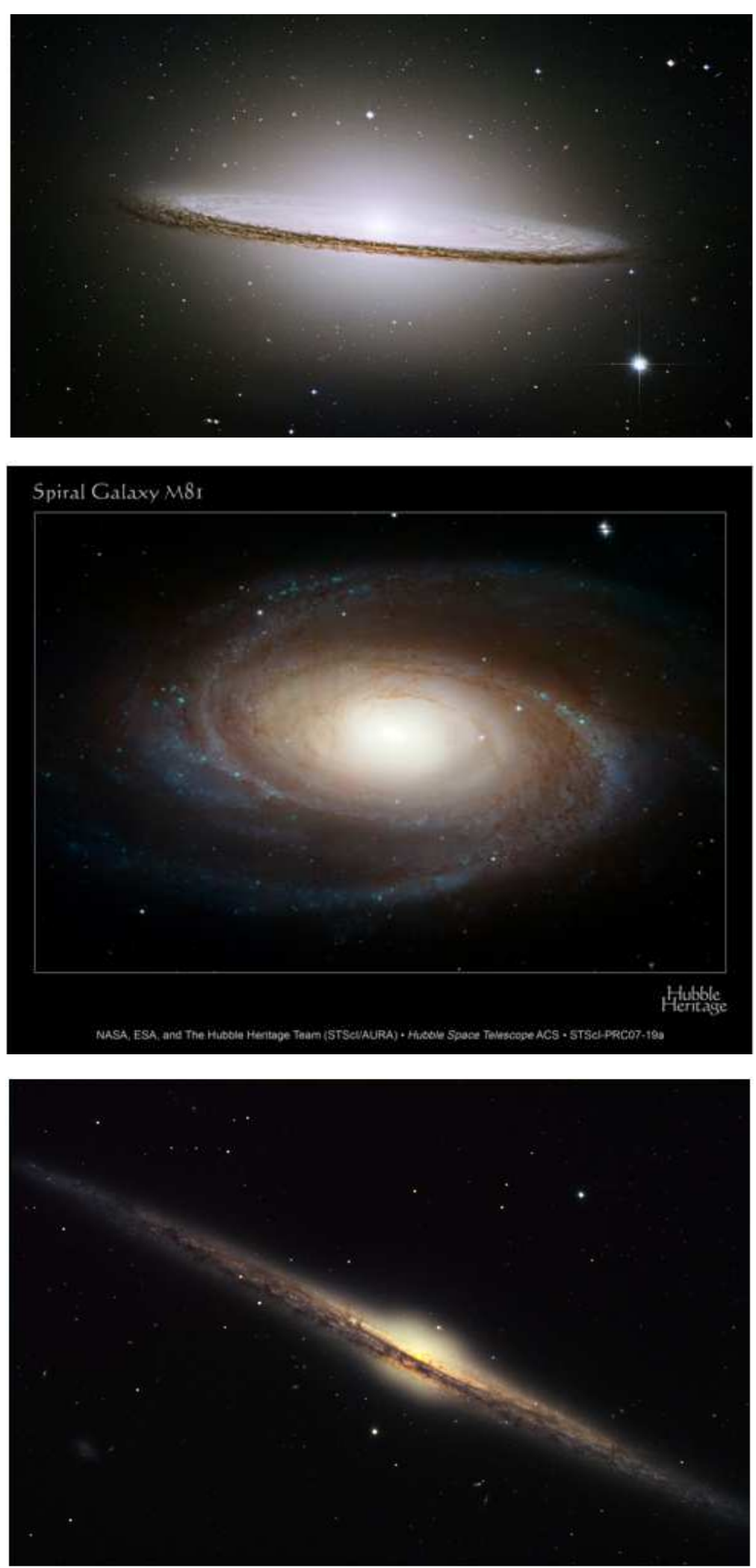

Fig. 1. Images (from top to bottom) of NGC 4594 (Sombrero or Messier Object 104) galaxy (en.wikipedia.org), NGC 3031 (Messier 81) galaxy (en.wikipedia.org) and NGC 4565 (Needle) galaxy (www.noao.edu), which show the common round thin-disk structure of spiral galaxies with small, amorphous, centrally located bulge. 
where, $V(r)$ is measured in units of a characteristic rotational velocity $V_{0}$.

As shown in our previous publications (Gallo and Feng, 2009; 2010; Feng and Gallo, 2011; 2014), equating (3) and (4) with slight algebraic arrangements yields a force-balance equation

$\int_{0}^{1}\left[\frac{E(m)}{\hat{r}-r}-\frac{K(m)}{\hat{r}+r}\right] \rho(\hat{r}) h \hat{r} d \hat{r}+\frac{1}{2} A V(r)^{2}=0$

where, $K(m)$ and $E(m)$ denote the complete elliptic integrals of the first kind and second kind, with:

$$
m \equiv \frac{4 \hat{r} r}{(\hat{r}+r)^{2}}
$$

The dimensionless parameter $A$ in (5), called the galactic rotation number, is defined as

$A \equiv \frac{V_{0}^{2} R_{g}}{M_{g} G}$

where, can be determined by introducing a constraint equation for mass conservation,

$2 \pi \int_{0}^{1} \rho(\hat{r}) h \hat{r} d \hat{r}=1$

\section{Gravitational Mass}

The integral (3) is equivalent to the left side of (2) but for a distributed mass in the galactic disk, while (4) has the same physical meaning as the right side of (2). With a readily measured rotation curve---the orbital velocity as a function of galactocentric radius, $V(r)$---the mass distribution in a galaxy can be determined by solving for $\rho(r)$ and $A$ in (5) and (7), as elaborated in our previous publications (Gallo and Feng, 2009; 2010; Feng and Gallo, 2011; 2014). The mass determined with this method is fundamentally the same as that described by (2) for determining the solar mass, although computing $\rho(r)$ in (5) is much more involved than calculating $M_{\text {sun }}$ from (2). It is sometimes called the "gravitational mass" (by people who care to make the distinction) as being derived from the gravitational field. (The measured rotation curve has been considered to provide the most reliable information for deriving the mass distribution in disk galaxies, according to Toomre, 1963; Sofue and Rubin, 2001).

With several computed examples from various types of measured rotation curves (e.g., Fig. 2), Feng and Gallo (2011; 2014) illustrated that most surface mass density profiles $\rho(r) h$ (with the abruptly varying ends at $r=0$ and 1 being trimmed out) exhibit approximately a common exponential law of decay, qualitatively consistent with the observed surface brightness distributions in spiral galaxies. As used for determining the solar mass from (2), Newtonian dynamics can describe the mass distribution in spiral galaxies self-consistently, according to the measured rotation curves. Therefore, we believe spiral galaxies described in this way are the rotating thin-disk galaxies through the eyes of Newton (Feng and Gallo, 2010). (The method described here should not be confused with the much simplified approach often described in textbooks and literature using the formula based on Keplerian dynamics, which can lead to erroneous results for disk galaxies, cf. Feng and Gallo, 2010).

\section{Luminous Mass}

Taken at face value, "luminous mass" is the mass of an object that is luminous and one can see. In astronomy, observable information is carried by "light"--electromagnetic radiation---emitted from the visible objects. Light can be analyzed to provide understanding about the emitting objects, such as their material constituents, surface temperature, distance, moving velocity, etc. But to derive the amount of mass in an object from the light it emits does not seem to fit the common sense based on our everyday life experiences. In fact, objects that do not emit light (and that can hardly be seen especially when far away) are quite common.

Asteroids, for example, are rocky objects revolving around the sun that are too small to be called planets. They do not emit light and are often hardly visible. Even identifying asteroids has barely begun in the $21 \mathrm{st}$ century, because they are not easily seen but are known to be abundant and of great threat to our existence. Only a guess based on extrapolating from cratering rates on the Moon suggested that some two thousand asteroids big enough to imperil civilized existence regularly cross the Earth's orbit. For those that had been identified (or seen), the values for their mass given on the NASA website in an Asteroid Fact Sheet (http://nssdc.gsfc.nasa.gov/planetary/factsheet/asteroidfa ct.html) are noted as only rough estimates, which offers a hint about the level of certainty in measuring mass on visible objects not even too far from the Earth in grant astronomical length scale.

Because astronomical observations rely on light, the luminosity measurements of galaxies among other objects have been extensively refined and analyzed in one or more specified wavelength bands (Binney and Tremaine, 2008). In the late $1950 \mathrm{~s}$, a systematic survey of the luminosity in spiral galaxies led De Vaucouleurs (1958) to establish the universal 'exponential disk' description of the radial surface brightness distribution in galactic disks. To derive the luminous mass from measured luminosity, the so-called mass-to-light ratio $M / L$ (in units of the solar value) has been used. But to determine the value of $M / L$, the value of mass density (for $M$ ) in addition to the measured luminosity density is needed. Although the local mass density may in principle be derived based on observed velocity dispersion perpendicular to the disk (in the z-direction) for a homogeneous stellar population (Oort, 1932; 1965), the certainty in measuring the galactic acceleration gradient perpendicular to the disk plane has proved elusive (Faber and Gallagher, 1979). 

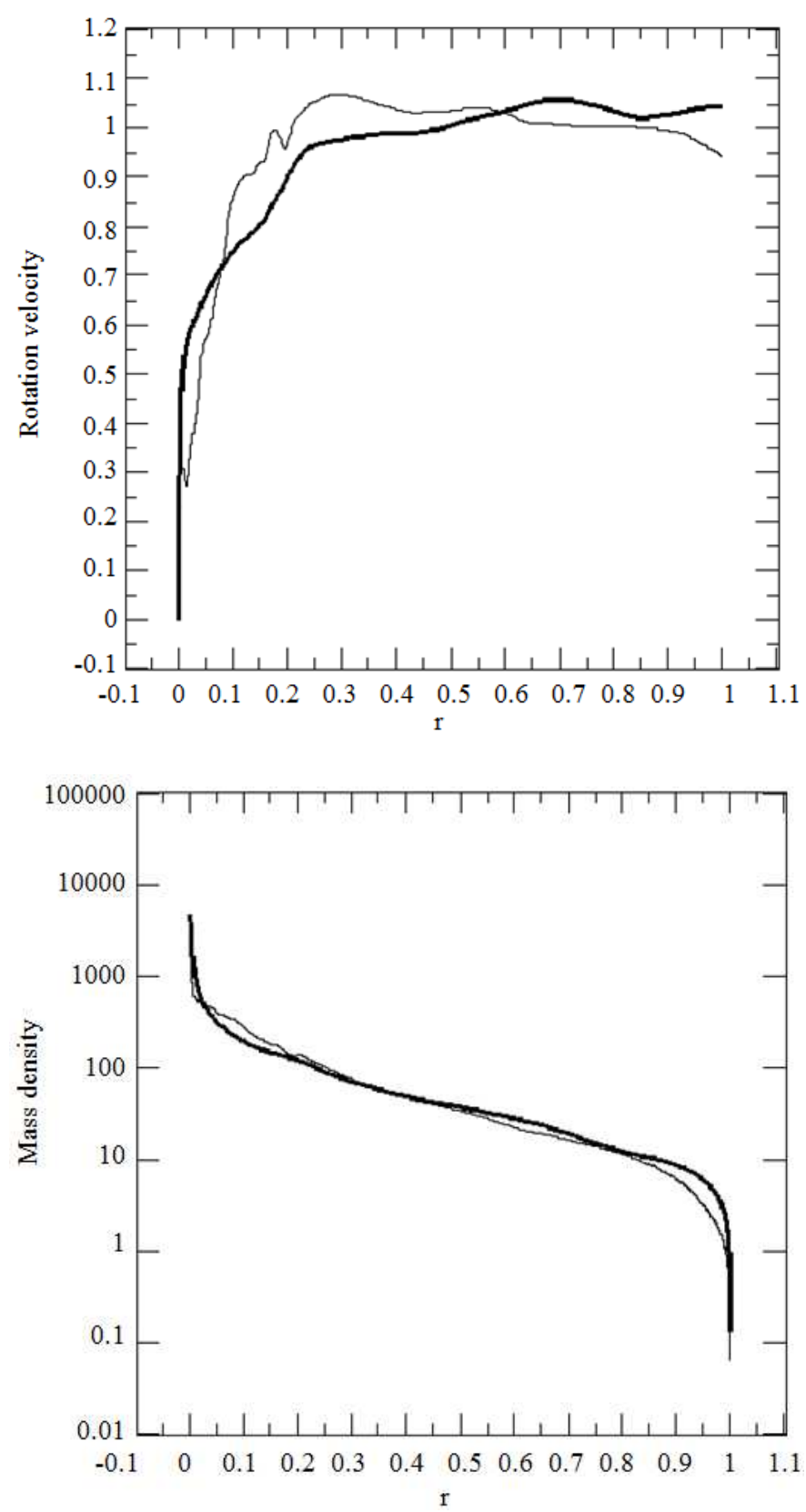

Fig. 2. Profiles of measured rotation velocity V(r) and computed mass density $\rho(r)$ for galaxies NGC $2403(\mathrm{Rg}=19.70 \mathrm{kpc}$, V0 $=$ $130 \mathrm{~km} / \mathrm{s}, \mathrm{A}=1.4918)$ and NGC $3198(\mathrm{Rg}=31.05 \mathrm{kpc}, \mathrm{V0}=160 \mathrm{~km} / \mathrm{s}, \mathrm{A}=1.6022)$, assuming $\mathrm{h}=0.01$ (Feng and Gallo, 2014). A large portion of $\rho(r)$, e.g., for $0.1<\mathrm{r}<0.9$, can be well approximated with a straight line in the semi-log plot, indicating that the mass density of most spiral galaxies follows a common exponential law of decline as qualitatively consistent with typical luminosity measurements.

Even for the main-sequence stars, which are typically believed as having the most reliable mass-luminosity relation, the scattering of data appears to be easily over orders of magnitude in the Hertzsprung-Russell diagram. Hence, considerable uncertainties are expected in cited values of $M / L$ shown in literature.

The intrinsic unreliability in estimating masses of galaxies from luminosity was pointed out by
Zwicky (1937), who has been credited for one of the first to use the term "dark matter." Because of the presence of the dark matter (which doesn't emit light but can absorb light to some unknown extent), masses estimated from observed luminosities are expected to be incomplete and can at best "furnish only the lowest limits" for the actual values (Zwicky, 1937). Based on Zwicky's reasoning, it should not be surprising to have 
large values of $M / L$ because not all objects containing mass can be luminous and seen.

An examination of the methods for determining the " $M$ " in $M / L$ (as discussed in Methods for Determining Mass) reveals the fact that $M$ of an astronomical object (such as the main-sequence stars) must come from the gravitational mass calculation (e.g., in the binary star systems). Hence, the luminous mass referred to in literature cannot be separated or obtained independently from the gravitational mass. It becomes clear that the luminous mass, if so preferred to term it, can be nothing more than a portion of the gravitational mass that encompasses both luminous and non-luminous (or "dark") mass. Finding that the luminous mass differ from (and especially less than) the gravitational mass is naturally expected and should not be surprising.

\section{Reasoning for Dark Matter}

The reasoning for dark matter in galaxies usually starts with the galactic rotation curve expected from the visible or luminous matter. Based on the apparent exponential decay of measured surface brightness in typical disk-like galaxies and an assumption of constant $M / L$, Freeman (1970) derived an elegant analytical formula for rotation curve $V(R)$ for the exponential disk from the given surface mass density $\mu(R)=\mu_{0} \exp (-\alpha R)$,

$$
V(R)^{2}=\pi G \mu_{0} \alpha R^{2}\left(I_{0} K_{0}-I_{1} K_{1}\right)
$$

where, $I_{\mathrm{n}}$ and $K_{\mathrm{n}}(\mathrm{n}=0$ and 1$)$ denote modified Bessel functions evaluated at $\alpha R / 2$. The rotation curve described by ( 8 ) indicates that the rotation velocity peaks at $\alpha R \approx 2.2$ (where $1 / \alpha$ is called the radial scale length) and then declines.

If a trustworthy value of $M / L$ could indeed be established, the surface mass density $\rho(r) h$ in (5) would then be simply obtained by multiplying the measured radial distribution of surface brightness distribution with $M / L$. Consequently, (5) can be used to predict the rotation curve $V(r)$ from a known $\rho(r) h$ (of arbitrary distribution not necessarily described by a simple exponential function) as demonstrated by Feng and Gallo (2011). Here, $\rho(r) h$ should be considered as the luminous surface mass density because it comes from the luminosity measurement.

\section{Flat H I Rotation Curves}

Until the early 1970 s, most galactic rotation curves were measured with optical signals, which did not extend beyond the luminous regions. The optical rotation curves with limited extension appeared consistent with (8). Later, radio synthesis telescopes were constructed to enable measurements of the $21 \mathrm{~cm}$ wavelength signals emitted from neutral atomic hydrogen (H I) far beyond the starlight. Against the prediction from the luminous mass, the $\mathrm{H}$ I rotation curves often do not show a decline over large radial distance. Instead, the rotation velocity stays nearly constant out to the limits of measurable data---which is often referred to as the 'flat' rotation curve. It is the flat rotation curve extending far beyond luminous galactic disk, indicating considerable amount of mass existing outside the region where matter could be seen, that has been believed to provide the compelling evidence for a dark matter component to dominate the total mass of spiral galaxies.

So, there can be some kind of "dark" matter that does not emit much light in comparison with the Sun (having $M / L>>1$ ), to explain the difference between gravitational mass and luminous mass. Such dark matter was envisioned by those authors who initiated the term dark matter (Oort, 1932; Zwicky, 1937), which entailed no mystery and should not be surprising. The neutral atomic Hydrogen (H I) that enabled rotation curve measurement based on the $21 \mathrm{~cm}$ wavelength signals exists far beyond the optical disk. Each hydrogen atom carries $1.67 \times 10^{-24}$ (g) of mass. If mass outside the optical disk with starlight is considered dark, the mass associated with H I must belong to the dark matter; its existence suggests the fact that the luminous mass alone cannot account for the mass of $\mathrm{HI}$ to realistically describe the galactic rotation behavior and not all of the observable mass (including $\mathrm{H}$ I) can be derived from luminosity. Simply put, the luminous mass cannot (and should not) be the same as the gravitational mass, as intuitively expected. There is nothing mysterious and puzzling about the frequently quoted discrepancy between gravitational mass and luminous mass.

\section{Non-baryonic Dark Matter}

However, the current popular belief (of serious scientists) is that the dark matter inferred from the difference between gravitational mass and luminous mass is mainly non-baryonic---the kind of matter other than the familiar protons and neutrons that make up stars and planets. This came from that a summation of the mass of all the baryonic matter, some of which could not be seen with the earlier optical telescopes but can now be detected in a myriad of new wavelengths with modern telescopes, still seems to fall "a long way short of accounting for the effects of dark matter" (Freeman and McNamara, 2006). Yet most of the estimates came from different techniques for studying the amount of matter at different scales with different types of uncertainties; "there is always the possibility that one or all of the estimates could be wrong" (Freeman and McNamara, 2006).

It is understandable that more sophisticated telescopes can enable seeing previously unseen (dark or dim) matter. But just seeing an object can hardly tell us the amount of mass it contains. For example, the radio 
synthesis telescopes can detect the neutral atomic hydrogen $(\mathrm{H} \mathrm{I})$ to enable measuring the $\mathrm{H}$ I rotation curves far beyond the optical disks. What this can tell us for sure is that there is definitely matter (such as H I) outside the optical disks, which was thought to be "dark" previously, now may not be dark anymore (because it can be seen). Atomic hydrogen is known to have mass, but its $M / L$, if could be determined with reasonable confidence, is very unlikely to have the same value as that of main-sequence stars. Although estimation of visible mass has been widely attempted (often with somewhat arbitrary assumptions on $M / L)$, the evaluation of associated uncertainty has rarely been seriously presented. To our understanding of the current measurement techniques, one should not be surprised by an order of magnitude uncertainty in most of the reported data.

Interestingly though, when Oort (1932) suspected dark matter in the Galactic disk by examining vertical stellar motions, it was estimated as "perhaps twice as much as was represented by the stars of the Milky Way"---suggesting only about a factor of two or three, which could very likely be regarded as a consequence of the unreliability in stellar mass data. But Oort's calculation was found to be erroneous by later scientists based on more sophisticated analysis; "the disk of the Milky Way---contrary to Oort's conclusion---is more or less free of dark matter." However, "Oort's 'discovery' of disk dark matter would in fact be a much more comfortable result" (probably for a desire of rounding up the apparently needed mass to explain the observed fast motions of surrounding globular clusters if they were assumed not to simply be passing through) (Freeman and McNamara, 2006).

By computing solutions of $\rho(r)$ to (5) from measured rotation curves $V(r)$, we found that up to the "cut-off" radius at $20.55(\mathrm{kpc})$ (beyond which there was no more detectable signal) a total mass of $1.41 \times 10^{11}\left(\mathrm{M}_{\text {Sun }}\right)$ is sufficient for supporting the Milky Way disk rotation (Feng and Gallo, 2014). This value is very close to the oft-quoted "about $10^{11}$ stars" in the Milky Way (Binney and Tremaine, 2008), in view of the appreciable uncertainties in astronomical mass calculations.

If we take $3.4 \times 10^{-20}\left(\mathrm{~kg} / \mathrm{m}^{3}\right)$ as the average gravitational mass density in the solar neighborhood (corresponding to $\sim 100\left(\mathrm{M}_{\mathrm{Sun}} / \mathrm{pc}^{2}\right)$, as predicted surface mass density needed at the solar galactocentric radius according to a measured rotation curve, with exact value in the range of $\sim 74$ to $144\left(\mathrm{M}_{\mathrm{Sun}} / \mathrm{pc}^{2}\right)$ depending on the size of bulge considered in a self-gravitating disk model (Feng and Gallo, 2014) and a disk thickness of 200 (pc), i.e., $h=0.01$ in (5), leading to an estimated volume mass density of $\sim 0.5\left(\mathrm{M}_{\mathrm{Sun}} / \mathrm{pc}^{3}\right)$, within the same order of magnitude as reported from other sources in Binney and Tremaine, 2008), there should be equivalently $\sim 2 \times 10^{7}$ hydrogen atoms in a cubic meter, or equivalently $\sim 20$ hydrogen atoms per $\mathrm{cm}^{3}$. This value falls well within the reported range of estimated gas density in the Interstellar Medium (ISM---the matter in the space between the stars in a galaxy, which fills interstellar space and blends smoothly into the surrounding intergalactic space) of our Galaxy (Ferriere, 2001; Binney and Tremaine, 2008). In terms of average mass density, the amount of matter in a galactic stellar system is extremely tenuous by terrestrial standards $\left(>10^{20}\right.$ atoms per $\left.\mathrm{cm}^{3}\right)$, even when the stars are included. Beyond the solar neighborhood, the mass density in a disk model is expected to further decrease nearly exponentially with galactocentric radius according to Newtonian dynamics and measured rotation curve (Feng and Gallo, 2011; 2014). Thus, the amount of mass required to support the observed rotation curve could be no more than that found in typical ISM.

Among the ISM, stars only form inside large complexes of cold molecular clouds, typically a few pc in size having a number density of $10^{2}--10^{6}$ molecules per $\mathrm{cm}^{3}$ and a fractional volume of $<1 \%$ (Ferriere, 2001). Because of the nearly exponential decrease of average mass density with galactocentric radius as shown in Figure 2, chances for star formation are expected to diminish beyond a certain galactocentric distance due to lack of dense molecular clouds where the ISM type of matter may still have relatively considerable amount of mass. Therefore, outside the optical disk where no more starlight can be seen, clouds of gas in ionic, atomic and molecular form and dust are likely to provide enough mass for explaining the observed flat rotation curve. Only a few (baryonic) atoms per $\mathrm{cm}^{3}$ in terms of the average number density could be sufficient. Is it really necessary to bring the non-baryonic dark matter in for explaining the observed flat rotation curves?

As of today, the basis for suggesting dominant amount of non-baryonic matter in galaxies is neither convincingly validated nor intuitively reasonable.

\section{Dark Matter Halo}

Moreover, the current popular model of a spiral galaxy consists of a decomposition of a bulge, a disk and a dark matter halo (Faber and Gallagher, 1979; Sofue and Rubin, 2001; Freeman and McNamara, 2006; Binney and Tremaine, 2008). The bulge---a small, amorphous, centrally located stellar system---and the disk with well-defined rotation curve are evidenced by optical observations (Fig. 1).

But the dark matter halo has no direct observational basis, with rather vague, brief explanations, if any, being provided in books and literature. According to several authors (Faber and Gallagher, 1979; Sofue and Rubin, 2001; Freeman and McNamara, 2006; Binney and Tremaine, 2008), the concept of dark matter halo sounded like coming from the N-body simulation of Ostriker and Peeble (1973), arguing that the most 
plausible way to stabilize the Galaxy against the bar instability was to add a massive dark halo. The dark halo was believed to provide part of the equilibrium gravitational field, thereby reducing the required disk mass and the destabilizing effect of the disk's self-gravity. But there are other more recent $\mathrm{N}$-body simulations showing that a disk galaxy with an almost flat rotation curve can be stabilized by dense centers without the dark matter halo (Sellwood and Evans, 2001), reversing the argument for requiring a massive halo to stabilize the disk. It was also suggested that halos are not very efficient for stabilizing the disk as compared to bulges (Kalnajs, 1987).

Yet still, the dark matter halos have been considered as an indispensable component of galaxies, despite that many authors tend to believe negligible mass contribution from the dark matter halo in the luminous disk region. This led to the so-called maximum disk hypothesis (van Albada and Sancisi, 1986), which assumes the mass of the luminous disk to be as large as possible to produce the galactic rotation curve, with the mass of the dark matter halo dominant in the outer region beyond the visible edge. But there is no clear explanation on why the dark matter halo takes a spherical shape and stays only in the outer region, except for the modeling convenience by assuming it as an isothermal sphere (Carignan and Freeman, 1985). Even to this day, "the shape of dark matter halos remains a mystery" (Freeman and McNamara, 2006). (From the modeling point of view, a pure thin disk based on a given rotation curve can yield a uniquely determined surface mass density distribution in a spiral galaxy; adding a spherical bulge or halo inevitably induces the requirements of known spherical mass distribution which, to our knowledge, can only come from debatable assumptions, as discussed by Feng and Gallo, 2014). Thus, the assumption of a dark matter halo containing substantial amount of mass lacks supporting evidence and logical rigor.

\section{Dark Matter in Bigger Pictures}

Outside the rotating Galactic disk, some randomly moving stars form a nearly spherical stellar halo. Those halo stars are "fast-moving, energetic stars, buzzing around the Galaxy like a swarm of bees" (Freeman and McNamara, 2006). Our galaxy also contains about 150 globular clusters---spherical collections of $10^{4}-10^{6}$ stars (Binney and Tremaine, 2008). Some of those halo stars or globular clusters may move at velocities so high that they exceed the estimated escape velocity of the Galaxy. If those fast moving objects were bound to the Galaxy by gravitation, much more Galactic mass than that to support the rotation curve would be needed. Thus, the idea of invisible dark matter could become entertaining. However, observations of the entire orbits of those randomly moving objects, which individually may take hundreds of million years to complete, can be extremely challenging. Without complete orbital knowledge about those halo stars, snapshots of their transient motions can hardly be taken as an observational evidence for dark matter.

In a larger scale, galaxies (or 'nebulae') appear to congregate in groups, called galaxy clusters, instead of being randomly distributed non-interacting loners in space. Zwicky (1937) investigated the Coma cluster by applying the virial theorem with a tentative hypothesis of "statistically stationary system." From the average velocity of observed galaxy motions, Zwicky estimated the kinetic energy of the system and found that the mass needed for the gravitational potential energy to prevent those galaxies from flying apart is about $4.5 \times 10^{13}\left(\mathrm{M}_{\text {Sun }}\right)$. With about 1000 galaxies in the Coma cluster, this led to the average mass of each galaxy to be about $4.5 \times 10^{10}$ $\left(\mathrm{M}_{\text {Sun }}\right)$, about one third of that of the Milky Way according to Feng and Gallo (2014), which was "somewhat unexpected" when compared to $8.5 \times 10^{7}$ $\left(\mathrm{M}_{\text {Sun }}\right)$ as suggested by the luminosity data. Therefrom, Zwicky has been credited as one of the first scientists for discovering the dark matter or missing mass. However, Zwicky in the same article was quite critical about the intrinsic unreliability in estimating galactic mass from its luminosity (which was rarely, if at all, mentioned in literature when talking about his first usage of the term dark matter, when he considered objects "in the form of cool and cold stars, macroscopic and microscopic solid bodies and gases.").

From a historical perspective, Zwicky was an admirably intelligent scientist who made many important contributions not only in astronomy but also in a wide range of other disciplines. He was one of the first to study galaxy clusters, through which he suggested serious discrepancy between gravitational mass and luminous mass which implied considerable amount of unseen or dark matter. However, using Zwicky's virial theorem calculations as the evidence for dark matter (especially the non-baryonic dark matter) seems to be too much of a stretch, in view of what we know about the level of uncertainties in estimating the luminous mass and counting the baryonic mass. In this regard, we should keep in mind that the volume of space between the stars and galaxies is tremendous; just miscounting a matter of a few atoms in a cubic centimeter in the interstellar medium, especially in the outer regions where the volume increases rapidly with the galactocentric radius, may easily lead to an order of magnitude difference in the estimated galactic mass.

When it comes to cosmology, the Big Bang Theory seems to be the present-day standard model of the origin of the Universe. It suggests that dark matter accounts for about $27 \%$ while ordinary (baryonic) matter accounts for only $4.9 \%$ of the mass-energy content of the observable universe, with the remainder being attributable to dark energy. Inasmuch as its acceptance as the current standard model, the Big Bang Theory has not been fully verified by the existing observational evidence and remains as a 
hypothetical theory. Alternative models have been proposed for our understanding of the Universe as a whole (Lopez-Corredoira, 2014). In fact, baryonic matter exists everywhere. Even in the vast expanses between the galaxies where it had always been assumed more or less empty, an entirely unsuspected source of stellar baryonic matter has recently been discovered (Freeman and McNamara, 2006).

\section{Conclusion}

When astronomers and astrophysicists found a difference or discrepancy between the gravitational mass and luminous mass in galaxies, they were puzzled and resorted to a mysterious matter that could not be seen--the dark matter. Some scientists would even consider such discrepancy as the compelling evidence for dark matter, as if the confidence level were quite high. However, little details could be found in quantifying the cascade of uncertainty in determining the luminous mass and gravitational mass, which is normally crucial when analyzing the difference between two quantities and establishing the confidence level thereof.

Estimating mass of an astronomical (or celestial) object far away is understandably challenging with many limitations in methods and tools. The consequence of the difficulties involved in measuring the distance of a star or a galaxy is a considerable level of uncertainty. Measuring the luminosity of a star depends on its distance which undoubtedly cascades its uncertainty to the luminosity calculation. Direct measurement of the mass of a star seems to be limited to the (eclipsing) binary star systems in the solar neighborhood, which depends on not only its distance but also the separation between the two stars. With the available data of mass and luminosity, an empirical mass-to-light ratio of a star can be obtained, which is then applied to estimating masses of other stars of the same type (i.e., sharing the similar color or surface temperature) whose direct mass measurement is intractable. Thus, the "luminous mass" determined from the luminosity and mass-to-light ratio can be at best a rather crude estimate, considering the aggregation and cascade of uncertainty involved in many converting steps based on assumptions not thoroughly verified. Of course, for each individual galaxy, derivation of its gravitational mass from the measured rotation curve also involves uncertainties associated with the measurements of stellar motion velocity and distance. But many observed spiral galaxies exhibit quite similar structural configurations and rotation characteristics, which at least offer some level of statistical confidence.

In view of the fact that all mass measurements of the astronomical objects are based on gravitational force, even that for estimating the mass-to-light ratio of a star which is in turn used to determine the luminous mass, the gravitational mass (which comes directly from gravitational effect measurement) is intuitively expected to be much more reliable than the luminous mass. In fact, the measured rotation curves have been considered to provide the most reliable means for determining the distribution of gravitational mass in spiral galaxies (Toomre, 1963; Sofue and Rubin, 2001). By closely examining the methods for evaluating the luminous mass, one can quickly realize that the so-called luminous mass can at best represent a portion of the gravitational mass and it is not supposed to match the gravitational mass. Therefore, it is natural to find less luminous mass than gravitational mass.

In general, the stellar systems such as galaxies are extremely tenuous in terms of average mass density. For example, our Newtonian dynamics model based on measured rotation curve predicted that the average (gravitational) mass density in the solar neighborhood is around $0.5\left(\mathrm{M}_{\mathrm{Sun}} / \mathrm{pc}^{3}\right)$ (Feng and Gallo, 2014) (or $10^{-20}$ $\left(\mathrm{kg} / \mathrm{m}^{3}\right)$. This corresponds to about 20 hydrogen atoms per $\mathrm{cm}^{3}$, well within the reported range of estimated mass density in the interstellar medium. Given the vast volume in a typical galaxy, a slight miscounting of matter due to observational limitations can cause huge variations in evaluation of the galactic mass. With this kind of perspective in mind, one would naturally wonder whether quantitative evaluation of the discrepancy between luminous mass and gravitational mass, especially for establishing the compelling evidence of missing mass, can really bear any meaningful fruits.

Actually the gravitational mass, as determined from a thin disk model with a given rotation curve according to Newtonian dynamics, can be quite reasonable when compared with the star counts (which relates to the luminous mass). For example, the Milky Way rotation curve with a cut-off radius at $20.55(\mathrm{kpc})$ leads to a total mass of $1.41 \times 10^{11}\left(\mathrm{M}_{\text {Sun }}\right)$, which is fairly close to the star counts of about 100 billion (Feng and Gallo, 2014), in view of the level of uncertainty in the stellar mass measurements. As far as the observed rotation curves are concerned, the corresponding gravitational mass distribution could be reasonably consistent with the distribution of luminosity if the assumption of a constant mass-to-light ratio is abandoned. And there is no concrete reason for having a constant mass-to-light ratio across the entire galaxy, except probably for the convenience of model calculations. If so, the existence of dark matter in galaxies may become baseless.

Dissatisfied with the inability of directly detecting the speculated non-baryonic dark matter, some scientists (e.g., Milgrom, 1983; Moffat, 2006) have resorted to theories for modifying the laws of classical mechanics. By using a metric-skew-tensor gravity based on Einstein's general relativity for modifying acceleration law, Brownstein and Moffat (2006) demonstrated capability of explaining observed galactic rotation curves without exotic dark matter. Yet, the validity of such 
modifications of Newtonian dynamics still depends on the certainty of the apparent discrepancy between luminous mass and (Newtonian) gravitational mass. Based on our present analysis, such an apparent discrepancy is likely a result of lack of understanding of the uncertainties in evaluating mass of a celestial object and of inadequate appreciation of the serious technical challenges in such an endeavor, rather than the reality.

In our opinion, a galaxy with star type distribution varying from its center to periphery may have a mass-tolight ratio varying accordingly depending on the galactocentric radius. Based on our thin-disk model computations from measured rotation curves, most galaxies have a typical mass density profile with a peak value at the galactic center falling rapidly within $\sim 5 \%$ of the cut-off radius and then declining nearly exponentially toward the edge (e.g., Fig. 2). Because the radial scale length for the exponentially declining portion is usually larger than that of the luminosity one, the mass-to-light ratio should increase with galactocenteric radius, corresponding to cooler and "darker" matter toward the periphery of the galactic disk as consistent with the edgeon view images of spiral galaxies (Fig. 1). The predicted mass density in the Galactic disk is well within the range of that observed interstellar medium and therefore can be considered reasonable. This leads us to believe that ordinary baryonic matter can be sufficient for supporting the observed galactic rotation curves; speculation of large amounts of non-baryonic matter may be based on an ill-conceived discrepancy between gravitational mass and luminous mass which appears to be unjustified.

If we follow the same vein of thought, serious shortcomings of the arguments for dominant amounts of dark matter needed to hold a galaxy clusters together and the like, are readily revealed. Our logical analysis presented here demonstrates a philosophical truth that one should not place too much faith in a reported result, no matter how eminent the scientist who presents it. Without doubt and skepticism, science cannot thrive and will stagnate.

\section{Funding Information}

The authors have no support or funding to report.

\section{Author's Contributions}

In the preparation of this manuscript, James Q. Feng took the responsibility of mathematical analysis and writing, while C.F. Gallo provided background knowledge and physical insights.

\section{Ethics}

This article is original and contains unpublished material. The corresponding author confirms that all of the other authors have read and approved the manuscript and no ethical issues involved.

\section{References}

Bennett, J., M. Donahue, N. Schneider and M. Voit, 2007. Cosmic Perspective: Stars, Galaxies and Cosmology. Pearson College Division, ISBN-01: 0805387579, pp: 380.

Brownstein, J.R. and J.W. Moffat, 2006. Galaxy rotation curves without nonbaryonic dark matter. Astrophys. J., 636: 721-741. DOI: 10.1086/498208

Binney, J. and S. Tremaine, 2008. Galactic Dynamics. Princeton University Press, 2nd Edn., Princeton Series in Astrophysics, Princeton, ISBN-10: 1400828724, pp: 904.

Carignan, C. and K.C. Freeman, 1985. Basic parameters of dark halos in late-type spirals. Astrophys. J., 294: 494-501. DOI: $10.1086 / 163316$

De Vaucouleurs, G., 1958. Photoelectric photometry of the Andromeda nebula in the UBV system. Astrophys. J., 128: 465-488. DOI: 10.1086/146564

Faber, S.M. and J.S. Gallagher, 1979. Masses and mass-to-light ratios of galaxies. Ann. Rev. Astronomy Astrophys., 17: 135-187. DOI: 10.1146/annurev.aa.17.090179.001031

Feng, J.Q. and C.F. Gallo, 2010. Rotating thin-disk galaxies through the eyes of Newton. arXiv:1007.3778.

Feng, J.Q. and C.F. Gallo, 2011. Modeling the Newtonian dynamics for rotation curve analysis of thin-disk galaxies. Res. Astronomy Astrophys., 11: 1429-1448. DOI: 10.1088/1674-4527/11/12/005

Feng, J.Q. and C.F. Gallo, 2014. Mass distribution in rotating thin-disk galaxies according to Newtonian dynamics. Galaxies, 2: 199-222. DOI: $10.3390 /$ galaxies2020199

Ferriere, K.M., 2001. The interstellar environment of our galaxy. Rev. Mod. Phys., 73: 1031-1066. DOI: 10.1103/RevModPhys.73.1031

Freeman, K.C. and G. McNamara, 2006. In Search of Dark Matter. 1st Edn., Illustrated, reprint, ISBN-10: 0387276165, pp: 158.

Freeman, K.C., 1970. On the disks of spiral and so Galaxies. Astrophys. J., 160: 811-860. DOI: $10.1086 / 150474$

Gallo, C.F. and J.Q. Feng, 2009. A thin-disk gravitational model for galactic rotation. Crisis Cosmol. Conf., 413: 289-303.

Gallo, C.F. and J.Q. Feng, 2010. Galactic rotation described by a thin-disk gravitational model without dark matter. J. Cosmol., 6: 1373-1380.

Kalnajs, A.J., 1978. Halos and disk stability. Proceedings of the IUA Symposium, Jun. 24-28, Princeton, Dordrecht, D. Reidel Publishing Co., pp: 289-296.

Lopez-Corredoira, M., 2014. Non-standard models and the sociology of cosmology. Stud. History Philosophy Mod. Phys., 46: 86-96. DOI: 10.1016/j.shpsb.2013.11.005 
Milgrom, M., 1983. A modification of the Newtonian dynamics as a possible alternative to the hidden mass hypothesis. Astrophys. J., 270: 365-370. DOI: $10.1086 / 161130$

Moffat, J.W., 2006. Scalar-tensor-vector gravity theory. J. Cosmol. Astroparticle Phys., DOI: 10.1088/1475-7516/2006/03/004

Oort, J.H., 1932. The force exerted by the stellar system in the direction perpendicular to the galactic plane and some related problems. Bulletin Astronom. Institute Netherlands, 6: 249-287.

Oort, J.H., 1965. Stellar dynamics. In: Galactic Structure, Blaauw, A. and M. Schmidt, (Eds.), Chicago University Press, Chicago, pp: 455-512.

Ostriker, J.P. and P.J.E. Peebles, 1973. A numerical study of the stability of flattened galaxies: Or, can cold galaxies survive. Astrophys. J., 186: 467-480. DOI: $10.1086 / 152513$
Sellwood, J.A. and N.W. Evans, 2001. The stability of disks in cusped potentials. Astrophys. J., 546: 176188. DOI: $10.1086 / 318228$

Sofue, Y. and V.C. Rubin, 2001. Rotation curves of spiral galaxies. Ann. Rev. Astronomy Astrophys., 39: 137-174. DOI: 10.1146/annurev.astro.39.1.137

Toomre, A., 1963. On the distribution of matter within highly flattened galaxies. Astrophys. J., 138: 385-392. DOI: $10.1086 / 147653$

Trimble, V., 1987. Existence and nature of dark matter in the universe. Ann. Rev. Astronomy Astrophys., 25: 425-472. DOI: 10.1146/annurev.aa.25.090187.002233

Van Albada, G.D. and R. Sancisi, 1986. Dark matter in spiral galaxies. Philosophical Trans. Royal Soc. London, 320: 447-464. DOI: $10.1098 /$ rsta. 1986.0128

Zwicky, F., 1937. On the masses of nebulae and clusters of nebulae. Astrophys. J., 86: 217-246. DOI: $10.1086 / 143864$ 